\title{
The Use of Virtual Surgical Planning for Management of Ameloblastoma: A Case Report
}

\author{
Akash Sivam $^{1 *}$, Yi Long Roy Ong ${ }^{1}$, Ankit Garg ${ }^{2}$ and Paul Sillifant ${ }^{2}$ \\ ${ }^{1}$ Registrar, Oral and Maxillofacial Surgery Unit, Royal Hobart Hospital, Hobart, Australia \\ ${ }^{2}$ Consultant, Oral and Maxillofacial Surgery Unit, Royal Hobart Hospital, Hobart, Australia
}

*Corresponding author: Akash Sivam, Oral and Maxillofacial Surgery Unit, Royal Hobart Hospital, Hobart, TAS 7000, Australia, Tel: (+61)-433229835

\begin{abstract}
Ameloblastoma is a rare progressively growing epithelial odontogenic neoplasm. Although benign, it can expand and invade surrounding structures, and to reoccur if not adequately removed. Current treatment is wide local excision with reconstruction. Traditional reconstructive options included autologous tissues, however, with the advent of virtual surgical planning and custom prosthesis surgeons can provide an increasingly precise functional and aesthetically pleasing reconstruction. The case presented highlights the use, application and outcomes of virtual surgical planning for reconstructing the mandible and temporomandibular joint post ameloblastoma resection.
\end{abstract}

\section{Keywords}

Ameloblastoma, Temporomandibular joint, Virtual surgical planning, Custom joint prosthesis

\section{Abbreviation}

TMJ: Temporomandibular Joint

\section{Introduction}

Ameloblastoma has more than a 100-year history of recognition and controversy. It has been described as "benign", "malignant" and "locally invasive." In the most recent World Health Organisation classification in 2017, Ameloblastoma was defined as a benign intraosseous progressively growing epithelial odontogenic neoplasm characterised by expansion and tendency for local recurrence if not adequately removed [1]. It is a rare neoplasm with an estimated annual incidence of 0.92 cases per million population per year but is the most common odontogenic tumour excluding odontomas $[1,2]$. Current treatment recommendation is wide local excision beyond radiographical margins with immediate reconstruction [3]. With the advent of virtual surgical planning, we now have a multitude of reconstructive options in our arsenal. Traditionally reconstructed with autologous options such as iliac crest, costochondral or fibular free flap, other newer options include custom prostheses that use virtual surgical planning and mirroring techniques to reconstruct a symmetrical jaw with much higher precision and aesthetic outcome [4]. If the lesion involves or is in close proximity to the temporomandibular joint (TMJ), the joint had to be excised and a custom TMJ reconstruction could be included in the prosthesis. We present a case of a patient with ameloblastoma, who had a resection that involved a reconstruction with a custom TMJ prosthesis.

\section{Case Report}

A 72-year-old Caucasian male was referred to our hospital after presenting to his dentist for routine dental care reporting pain in the left mandible region. He reported no other symptoms but had an obvious painless left facial swelling. His past medical history included Wolff-Parkinson-White Syndrome diagnosed in childhood and managed conservatively, a right total hip replacement eight years prior, osteoarthritis and hypertension. On examination he had a painless left mandibular swelling, no dental mobility or signs of infection, with normal trigeminal and facial nerve function.

Citation: Sivam A, Ong YLR, Garg A, Sillifant P (2022) The Use of Virtual Surgical Planning for Management of Ameloblastoma: A Case Report. Res Rep Oral Maxillofac Surg 6:062. doi.org/10.23937/26433907/1710062

Accepted: February 14, 2022 ; Published: February 16, 2022

Copyright: (C) 2022 Sivam A, et al. This is an open-access article distributed under the terms of the Creative Commons Attribution License, which permits unrestricted use, distribution, and reproduction in any medium, provided the original author and source are credited. 


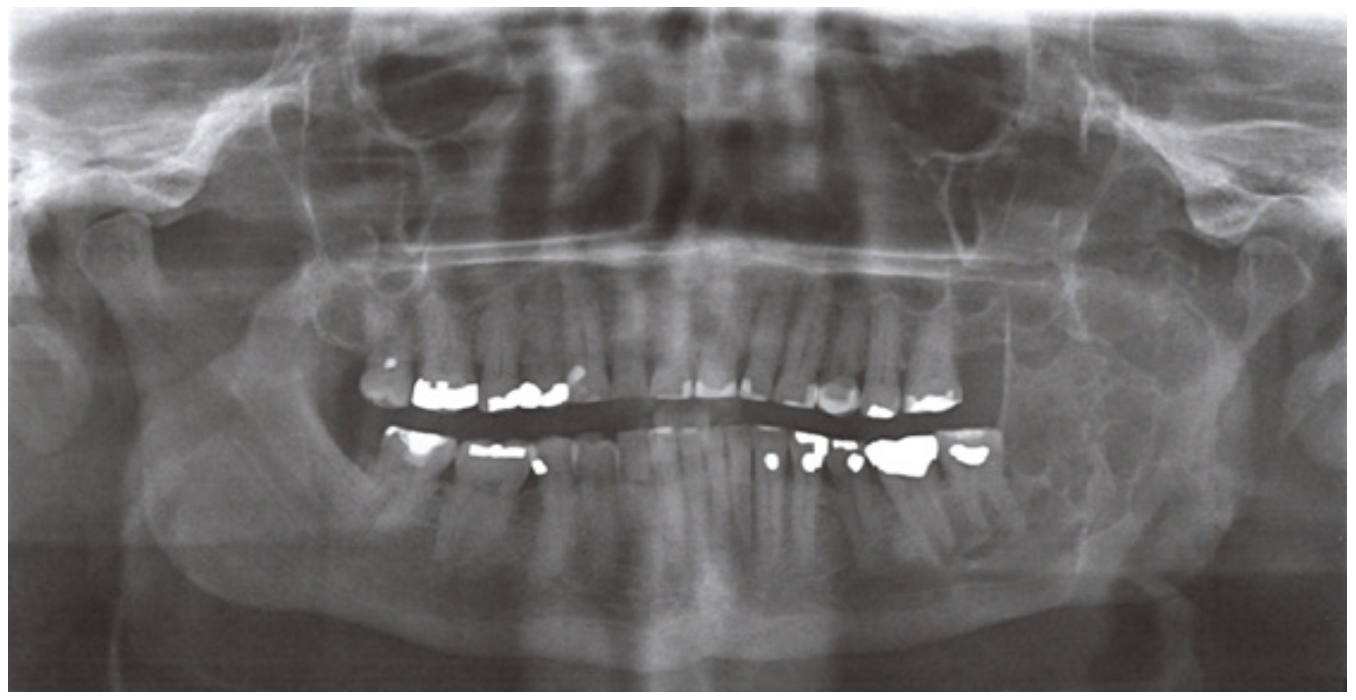

Figure 1: Panoramic radiographic view demonstrating multilocular radiolucency expansile lesion left mandible.

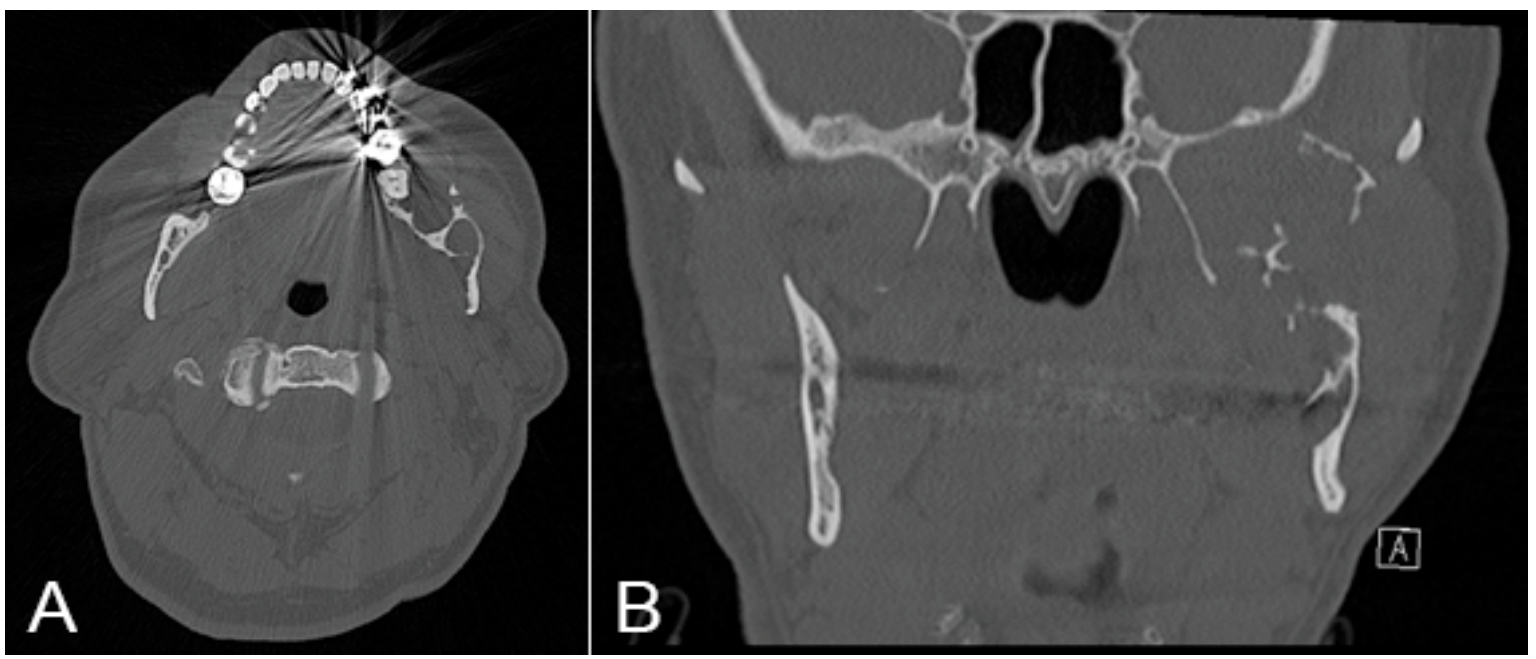

Figure 2: CT demonstrating expansile osteolytic mass (A) Axial view; (B) Coronal view.

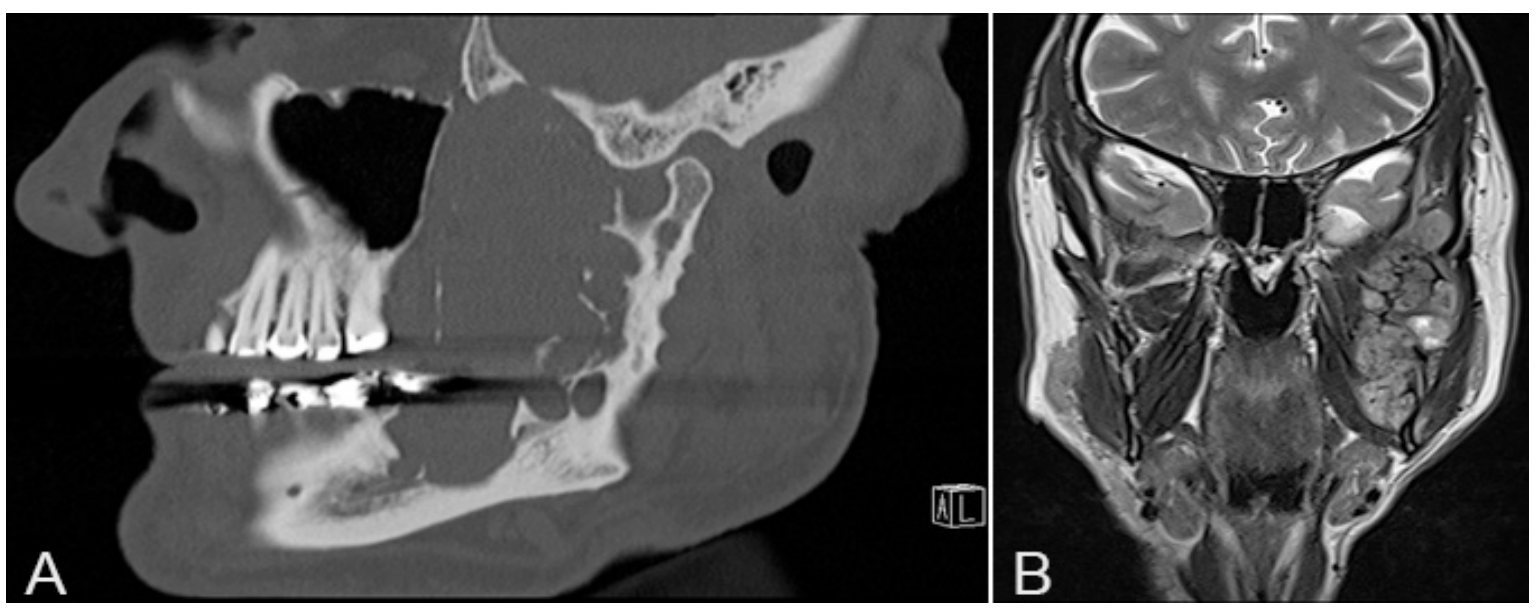

Figure 3: MRI demonstrating lesion (A) Sagittal view; (B) Coronal view.

Orthopantomogram (OPG) showed a multilocular radiolucency expansile lesion of the left mandibular body to condylar region (Figure 1).

Computed tomography (CT) demonstrated a large $77 \mathrm{~mm} \times 51 \mathrm{~mm} \times 27 \mathrm{~mm}$ expansile osteolytic mass involving the left mandibular ramus, extending to the left mandibular body with multiple foci of cortical destruction and involvement of the interior alveolar nerve (Figure 2). On magnetic resonance imaging (MRI) the lesion was heterogeneous in signal intensity on 
both $\mathrm{T} 1$ and $\mathrm{T} 2$-weighted series, with extension to the masticator space involving the masseter, temporalis, lateral and medial pterygoid muscles. It abutted the deep surface of the parotid gland with no evidence of direct gland involvement and no evidence of perineural infiltration (Figure 3).

A hard tissue biopsy was performed and histology showed nests of peripheral columnar cells with reverse polarisation and surrounding areas of stellate reticulum like cells, within a fibrous tissue stroma. There was rare mitotic activity and no evidence of significant cytologic atypia or necrosis. There was no lymphovascular or perineural invasion. Features were suggestive of ameloblastoma.

Due to the extent of the lesion, and to achieve adequate margins, resection would involve clearance of the infratemporal fossa and the left hemimandible, including the TMJ. Virtual surgical planning was conducted utilising high resolution computed tomography (CT) and the OMX solutions platform.

A custom mandible guide for use before and after resection was fabricated that included drilling guide holes for 3 inferior screws in Custom Condyle
Component with $2.7 \mathrm{~mm}$ diameter drilling guide holes and a cutting guide (Figure 4).

A custom fossa guide was fabricated with drilling guide holes for all screws in the custom fossa with 2.3 $\mathrm{mm}$ diameter drilling guide holes (Figure 5).

A custom fossa component was fabricated made with ultra-high molecular weight polyethylene to be used with $4 \times 2.0 \mathrm{~mm}$ screws (Figure 6).

A custom left condyle component made with titanium spanning the resected ramus, angle and body was fabricated with a rough bone contacting surface, for $4 \times 2.5 \mathrm{~mm}$ screws (Figure 7 ).

Below were 3 dimensional reconstructions of preop and expected post-op placement of the prosthesis (Figure 8).

A left-hand side hemi-mandibulectomy for resection of ameloblastoma and custom TMJ reconstruction was performed under general anaesthesia. A left modified pre-auricular, extended submandibular and intraoral incisions, and left zygomatic arch osteotomy was performed to gain access to the infratemporal fossa and hemimandible. The facial nerve was protected.
A

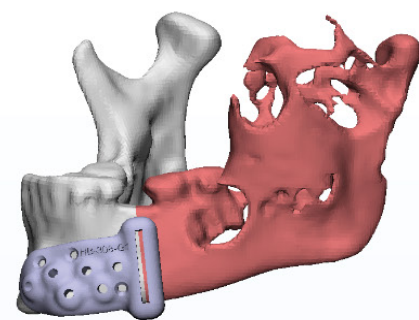

B

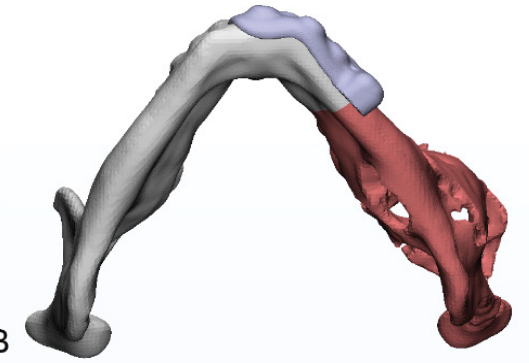

C

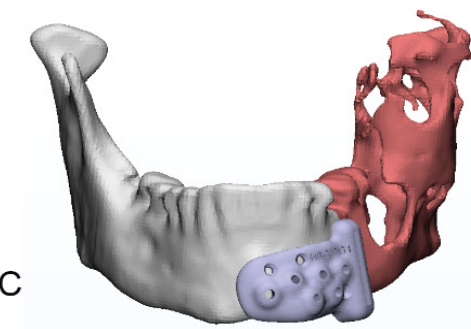

$\mathrm{F}$

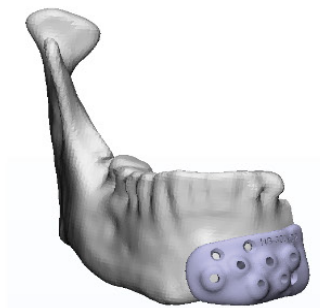

Figure 4: Custom condyle component $(A, D)$ Lateral view; $(B, E)$ Inferior view; $(C, F)$ Anterior view.

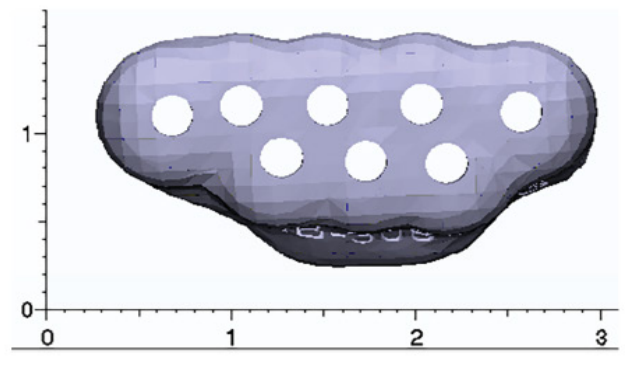

A

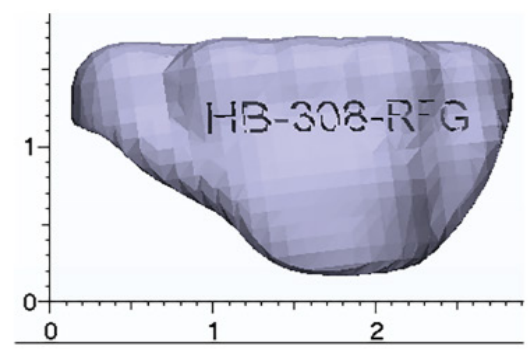

B

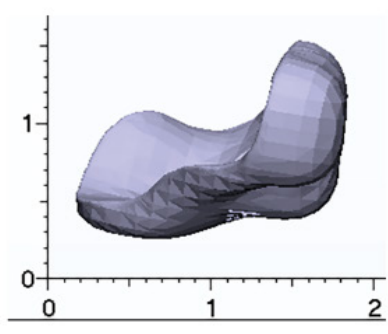

C

Figure 5: Custom fossa guide (A) Lateral view; (B) Inferior view; (C) Anterior view. 

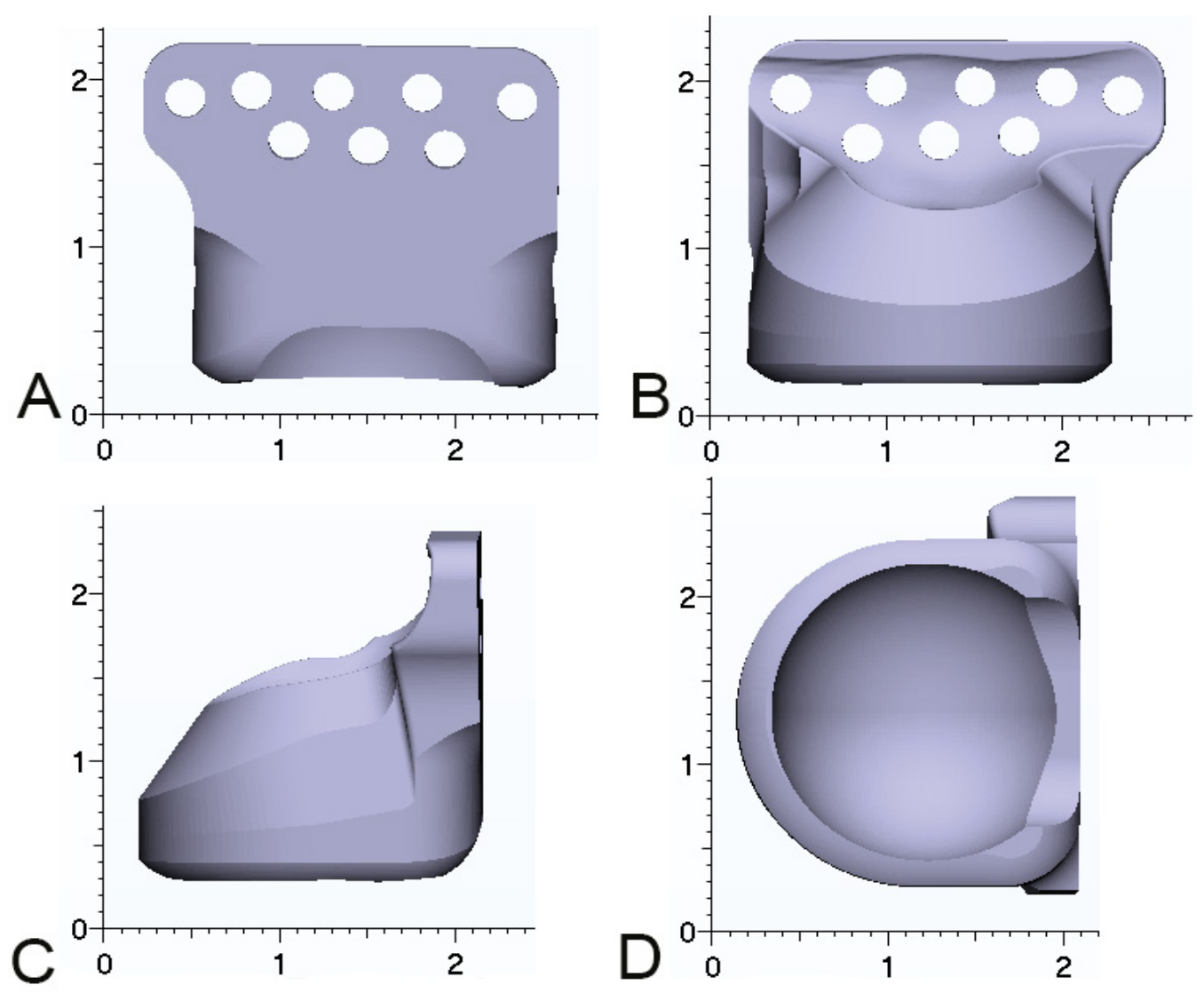

Figure 6: Custom fossa component (A) Lateral view; (B) Medial view; (C) Anterior view; (D) Inferior view.

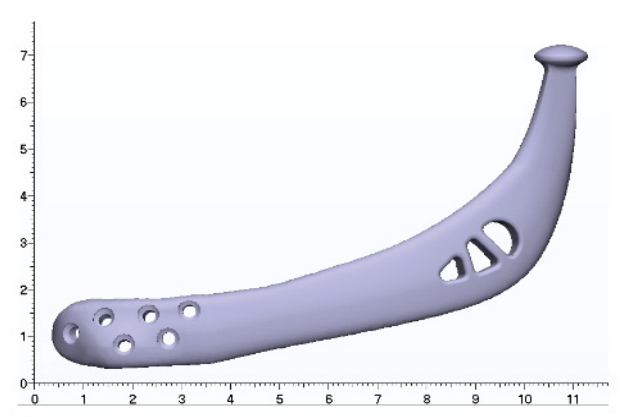

A

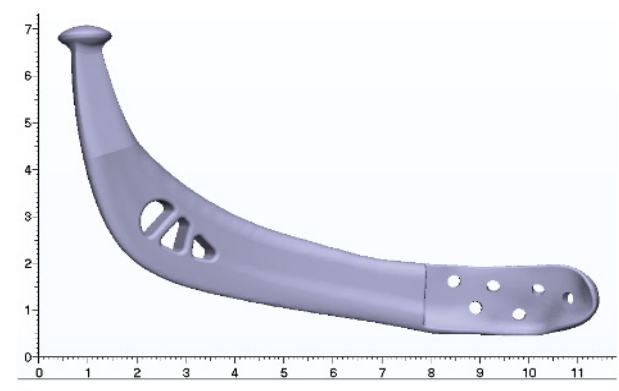

B

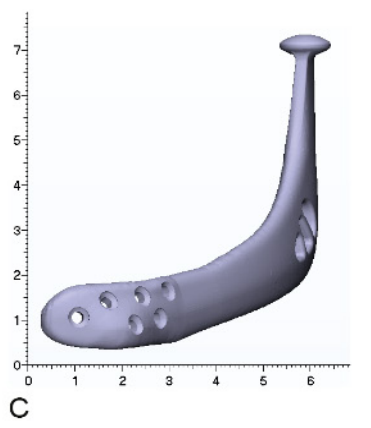

C

Figure 7: Custom left condyle component (A) Lateral view; (B) Medial view; (C) Anterior view.

Resection of the left mandible from parasymphysis to the fossa with $1.5 \mathrm{~cm}$ bony margins and soft tissue cuff of masseter, temporalis, pterygoids and mylohyoid was performed. The lingual nerve was preserved. The custom OMX Solutions TMJ concepts custom fossa and prosthesis was placed and secured with screws, followed by $2 \mathrm{~g}$ cephazolin irrigation of prosthesis. Occlusion arch bars with guiding elastics were placed to ensure proper occlusion. Wounds were closed with 3-0 chromic intra-oral, 3-0 vicryl deep sutures, 3-0 monocryl subcutaneous sutures and staples to scalp. No intra-operative complications were encountered during surgery.

Post-operatively he was initially managed in the intensive care unit (ICU) overnight before transfer to the ward. He was administered IV cephazolin and metronidazole and was fed via a nasogastric (NG) tube while he was nil by mouth for five days. By day five postoperatively he was tolerating a nourishing fluid diet and his NG tube was removed. He was then discharged home day six post-surgery. Figure 9 demonstrates immediate post-operative PA mandible showing placement of prosthesis.

At three weeks post-operatively he noted weakness in his left marginal mandibular branch of facial nerve and a maximal mouth opening of $25 \mathrm{~mm}$. He was advised to continue a puree diet for six weeks, at which point he started to upgrade his diet as tolerated. Six months post-operative CT demonstrated good positioning of prosthesis and no evidence of recurrence (Figure 10). Eight months post-operative MRI showed no sign of residual or recurrent tumour. At one year post-surgery 


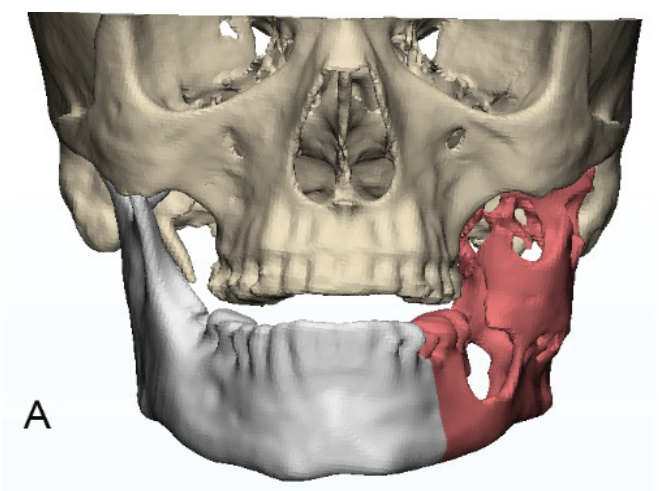

B
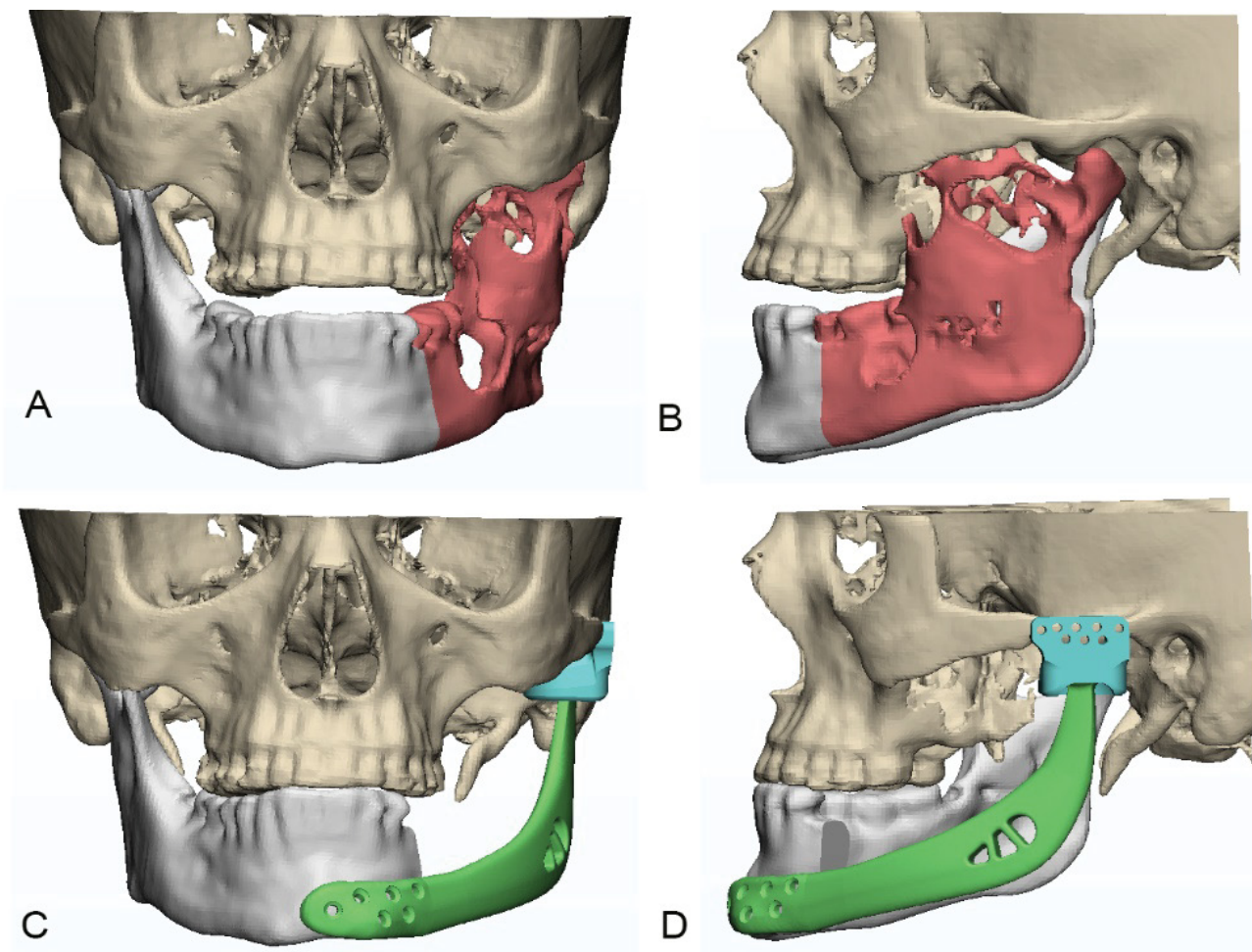

D

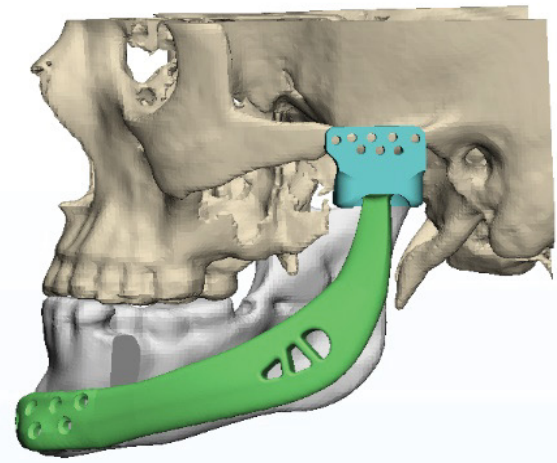

Figure 8: Three-dimensional (3D) reconstructions of prosthesis (A,B) Pre-op placement of the prosthesis; (C,D) Post-op placement of the prosthesis.

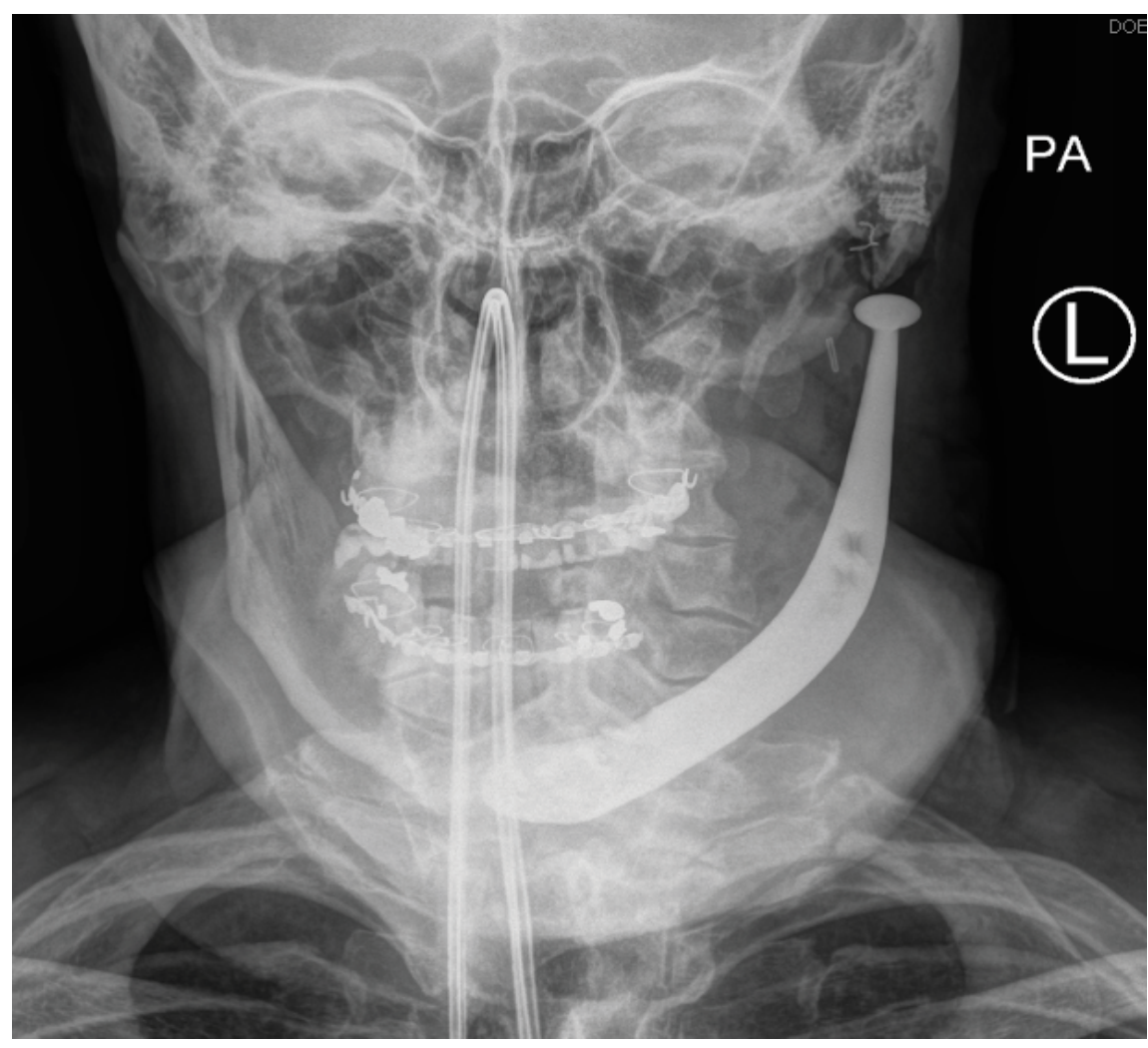

Figure 9: Post-operative PA Mandible.

his maximal mouth opening had improved to $30 \mathrm{~mm}$ and he had ongoing House-Brackmann grade II left marginal mandibular nerve weakness.

\section{Discussion}

TMJ total joint replacements using alloplastic materials have multiple benefits such as lack of donorsite morbidity, less prone to deterioration, lack of dimensional changes and therefore stable occlusion, ability to manipulate prosthesis design, maintain vertical height of ramus and prevent jaw deviation, reduced operative time, and return to immediate function 


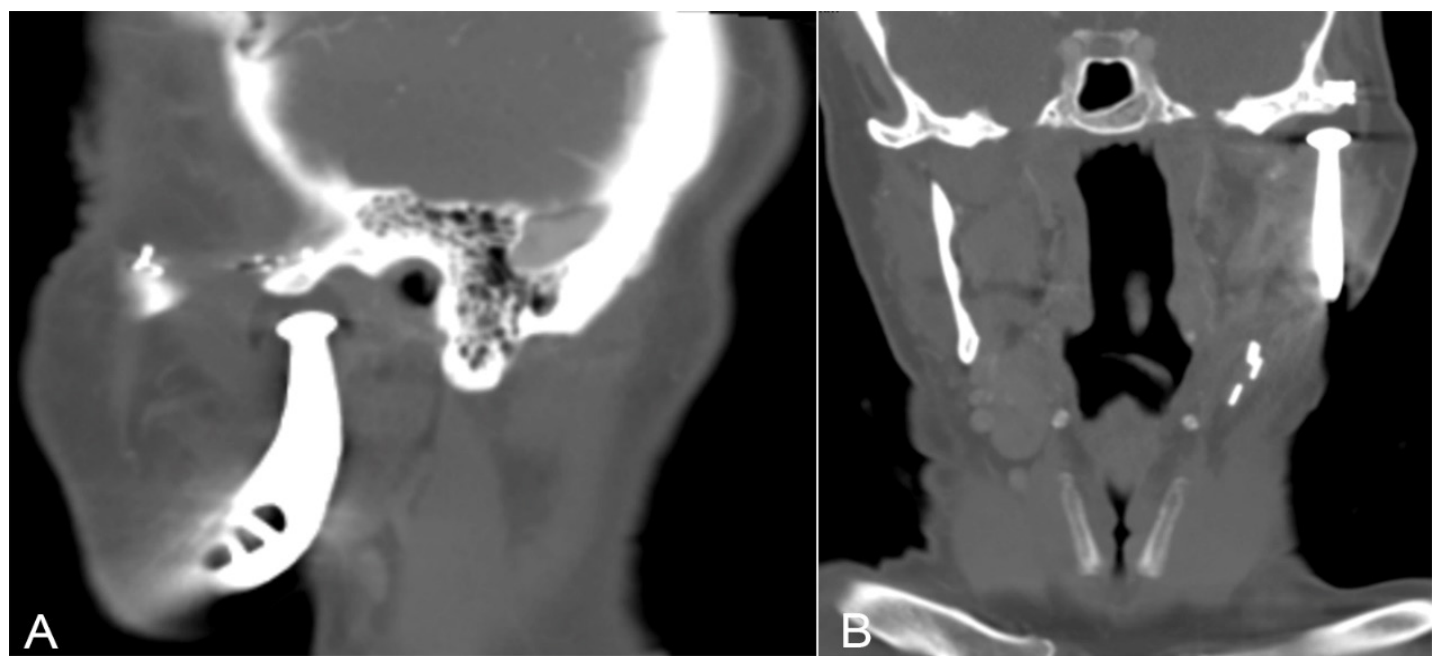

Figure 10: Six months post-operative CT (A) Lateral view; (B) Front view.

[5]. There are currently two options for alloplastic materials; stock prothesis that are contoured to the patient during surgery, and custom-made implants that are individually made for each patient $[6,7]$. Because this patient had a wide local excision, a custom-made prosthesis was chosen to incorporate the resected ramus, angle and body. A potential disadvantage of using a custom prosthesis is the cost and lead time to receiving the components.

The alternative option of autologous reconstruction has the benefit of biological compatibility, growth potential in children, and dental rehabilitation with use of dental implants and prosthesis $[6,8]$. Disadvantages are the risk of unpredictable growth of the grafts, the donor-site morbidity, altered resorption of the grafts, and risk of reankylosis $[6,9,10]$. Furthermore, to encourage blood supply to the graft to promote incorporation and reduce risk of failure, function such as early mobility of the joint is delayed and there is a risk of reanklyosis [11]. These risks have led to the use of alloplasts as interpositional materials.

Custom implants appear to provide stable and improved long-term outcomes over stock and autologous implants [12]. A custom implant is designed to fit the patient's bone, making it more predictable therefore reducing operative time, and the design can be mirrored to the contralateral side, with improved symmetry and aesthetics. Additionally, by virtue of being able to change the anteroposterior and vertical dimensions, custom implants allow changes to be made to occlusion [12].

\section{Conclusions}

This case study showed the steps and thought processes in a reconstruction of a hemi-mandibular defect post ameloblastoma resection with custom prosthesis and TMJ replacement. This is a viable option with today's technology in virtual surgical planning with good functional and aesthetic outcome.

\section{Acknowledgement}

The authors thank Maxoniq Pty Ltd. for proving high resolution images.

\section{Competing Interest}

The authors report no competing interests.

\section{Funding}

No funding was received for this study.

\section{Authors Contribution}

All authors contributed equally and approve submission of the manuscript for publication.

\section{References}

1. El-Naggar AK, Chan JKC, Grandis JR, Takata T, Slootweg PJ (2017) WHO classification of head and neck tumours, $4^{\text {th }}$ edition. International Agency for Research on Cancer 9.

2. Hendra FN, Van Cann EM, Helder MN, Ruslin M, de Visscher JG, et al. (2020) Global incidence and profile of ameloblastoma: A systematic review and meta-analysis. Oral Dis 26: 12-21.

3. McClary AC, West RB, McClary AC, Pollack JR, Fischbein NJ, et al. (2016) Ameloblastoma: A clinical review and trends in management. Eur Arch Otorhinolaryngol 273: 1649-1661.

4. Sembronio S, Tel A, Costa F, Isola M, Robiony M (2019) Accuracy of custom-fitted temporomandibular joint alloplastic reconstruction and virtual surgical planning. Int J Oral Maxillofac Surg 48: 1077-1083.

5. Sarlabous M, Psutka DJ (2018) Treatment of mandibular ameloblastoma involving the mandibular condyle: Resection and concomitant reconstruction with a custom hybrid total joint prosthesis and iliac bone graft. J Craniofac Surg 29: e307-e314.

6. Mani B, Balasubramaniam S, Balasubramanian $S$, Jayaraman B, Thirunavukkarasu R (2020) Role of custommade prosthesis for temporomandibular joint replacement in unilateral ankylosis - An evaluative study. Ann Maxillofac Surg 10: 344-352.

7. Movahed R, Mercuri LG (2015) Management of 
temporomandibular joint ankylosis. Oral Maxillofac Surg Clin North Am 27: 27-35.

8. Resnick CM, Genuth J, Calabrese CE, Taghinia A, Labow $\mathrm{BI}$, et al. (2018) Temporomandibular joint ankylosis after ramus construction with free fibula flaps in children with hemifacial microsomia. J Oral Maxillofac Surg 76: 2001.e12001.e15.

9. Hu YH, Zhang LZ, He DM, Yang C, Chen MJ, et al. (2017) Simultaneous treatment of temporomandibular joint ankylosis with severe mandibular deficiency by standard TMJ prosthesis. Sci Rep 7: 45271.
10. Marx RE (2007) Bone and Bone Graft Healing. Oral Maxillofac Surg Clin North Am 19: 455-466.

11. Johnson NR, Roberts MJ, Doi SA, Batstone MD (2017) Total temporomandibular joint replacement prostheses: A systematic review and bias-adjusted meta-analysis. Int $\mathrm{J}$ Oral Maxillofac Surg 46: 86-92.

12. Elledge R, Mercuri LG, Attard A, Green J, Speculand B (2019) Review of emerging temporomandibular joint total joint replacement systems. Br J Oral Maxillofac Surg 57: 722-728. 